\title{
The Congruence Subgroup Problem for finitely generated Nilpotent Groups
}

\author{
David El-Chai Ben-Ezra, Alexander Lubotzky
}

May 8, 2020

\begin{abstract}
The congruence subgroup problem for a finitely generated group $\Gamma$ and $G \leq A u t(\Gamma)$ asks whether the map $\hat{G} \rightarrow A u t(\hat{\Gamma})$ is injective, or more generally, what is its kernel $C(G, \Gamma)$ ? Here $\hat{X}$ denotes the profinite completion of $X$. In the case $G=A u t(\Gamma)$ we denote $C(\Gamma)=C(A u t(\Gamma), \Gamma)$.

Let $\Gamma$ be a finitely generated group, $\bar{\Gamma}=\Gamma /[\Gamma, \Gamma]$, and $\Gamma^{*}=\bar{\Gamma} / \operatorname{tor}(\bar{\Gamma}) \cong$ $\mathbb{Z}^{(d)}$. Denote

$$
A u t^{*}(\Gamma)=\operatorname{Im}\left(\operatorname{Aut}(\Gamma) \rightarrow \operatorname{Aut}\left(\Gamma^{*}\right)\right) \leq G L_{d}(\mathbb{Z}) .
$$

In this paper we show that when $\Gamma$ is nilpotent, there is a canonical isomorphism $C(\Gamma) \simeq C\left(A u t^{*}(\Gamma), \Gamma^{*}\right)$. In other words, $C(\Gamma)$ is completely determined by the solution to the classical congruence subgroup problem for the arithmetic group $A u t^{*}(\Gamma)$.

In particular, in the case where $\Gamma=\Psi_{n, c}$ is a finitely generated free nilpotent group of class $c$ on $n$ elements, we get that $C\left(\Psi_{n, c}\right)=C\left(\mathbb{Z}^{(n)}\right)=$ $\{e\}$ whenever $n \geq 3$, and $C\left(\Psi_{2, c}\right)=C\left(\mathbb{Z}^{(2)}\right)=\hat{F}_{\omega}=$ the free profinite group on countable number of generators.
\end{abstract}

Mathematics Subject Classification (2010): Primary: 19B37, 20F18; Secondary: 20H05, 20E $36,20 \mathrm{E} 18,11 \mathrm{H} 56,20 \mathrm{~F} 40$.

Key words and phrases: congruence subgroup problem, nilpotent groups, automorphism groups, profinite groups.

\section{Introduction}

Let $G \leq G L_{n}(\mathbb{Z})$. The classical congruence subgroup problem (CSP) asks whether every finite index subgroup of $G$ contains a principal congruence subgroup, i.e. a subgroup of the form $G(m)=\operatorname{ker}\left(G \rightarrow G L_{n}(\mathbb{Z} / m \mathbb{Z})\right)$ for some $0 \neq m \in \mathbb{Z}$. Equivalently, it asks whether the natural map $\hat{G} \rightarrow G L_{n}(\hat{\mathbb{Z}})$ is injective, where $\hat{G}$ and $\hat{\mathbb{Z}}$ are the profinite completions of the group $G$ and the $\operatorname{ring} \mathbb{Z}$, respectively. More generally, the CSP asks what is the kernel of this map. It is a classical $19 \underline{\underline{t h}}$ century result that for $G=G L_{n}(\mathbb{Z})$ the answer 
is negative when $n=2$. Moreover (but not so classical, cf. [Mel, [L1]), the kernel in this case is $\hat{F}_{\omega}$ - the free profinite group on a countable number of generators. On the other hand, it was proved in the sixties by Mennicke Men] and Bass-Lazard-Serre [BLS] that for $n \geq 3$ the map is injective, and the kernel is therefore trivial. This breakthrough led to a rich theory which studied the CSP for many other arithmetic groups. It has been solved for many arithmetic groups, but not yet for all. See Rag and Rap for surveys.

By the observation $G L_{n}(\mathbb{Z}) \cong A u t\left(\mathbb{Z}^{(n)}\right)$, the CSP can be generalized as follows: Let $\Gamma$ be a group and $G \leq A u t(\Gamma)$. For a finite index characteristic subgroup $M \leq \Gamma$ denote

$$
G(M)=\operatorname{ker}(G \rightarrow \operatorname{Aut}(\Gamma / M)) .
$$

Such a $G(M)$ is called a "principal congruence subgroup" and a finite index subgroup of $G$ which contains $G(M)$ for some $M$ is called a "congruence subgroup". The CSP for the pair $(G, \Gamma)$ asks whether every finite index subgroup of $G$ is a congruence subgroup.

One can see that the CSP is equivalent to the question: Is the congruence map $\hat{G}=\lim G / U \rightarrow \lim G / G(M)$ injective? Here, $U$ ranges over all finite index normal subgroups of $G$, and $M$ ranges over all finite index characteristic subgroups of $\Gamma$. When $\Gamma$ is finitely generated, it has only finitely many subgroups of a given index $m$, and thus, the charateristic subgroups $M_{m}=\cap\{\Delta \leq \Gamma \mid[\Gamma: \Delta]=m\}$ are of finite index in $\Gamma$. Hence, one can write $\Gamma=\varliminf_{m \in \mathbb{N}} \Gamma / M_{m}$ and have 1

$$
\begin{aligned}
\lim _{\leftarrow} G / G(M) & =\lim _{m \in \mathbb{N}} G / G\left(M_{m}\right) \leq \lim _{m \in \mathbb{N}} A u t\left(\Gamma / M_{m}\right) \\
& =\operatorname{Aut}\left(\varliminf_{m \in \mathbb{N}}\left(\Gamma / M_{m}\right)\right)=\operatorname{Aut}(\hat{\Gamma}) .
\end{aligned}
$$

Therefore, when $\Gamma$ is finitely generated, the CSP is equivalent to the question: Is the congruence map $\hat{G} \rightarrow A u t(\hat{\Gamma})$ injective? More generally, the CSP asks what is the kernel $C(G, \Gamma)$ of this map. For $G=\operatorname{Aut}(\Gamma)$ we will also use the simpler notation $C(\Gamma)=C($ Aut $(\Gamma), \Gamma)$.

The classical CSP results mentioned above can therefore be reformulated as $C\left(\mathbb{Z}^{(2)}\right)=\hat{F}_{\omega}$ while $C\left(\mathbb{Z}^{(n)}\right)=\{e\}$ for $n \geq 3$. Recently, it was proved that when $\Gamma=\Phi_{n}$ is the free metabelian group on $n$ generators, we have: $C\left(\Phi_{2}\right)=\hat{F}_{\omega}$, $C\left(\Phi_{3}\right) \supseteq \hat{F}_{\omega}$, and for every $n \geq 4, C\left(\Phi_{n}\right)$ is abelian (see $\left.\mathrm{Be} 1, \overline{\mathrm{Be} 2}, \mathrm{Be} 3, \mathrm{BL}\right)$. I.e. while in the free abelain case there is a dichotomy between $n=2$ and $n \geq 3$, in the free metabelian case we have dichotomy between $n=2,3$ and $n \geq 4$.

The goal of this paper is to show that contrary to the above metabelian cases, when $\Gamma$ is a finitely generated nilpotent group, the CSP for $\Gamma$ is completely determined by the CSP for abelian groups. Let us put things more precise: Let

\footnotetext{
${ }^{1}$ When we write $\operatorname{Aut}(\hat{\Gamma})$ we basically mean to consider the group of continuous automorphisms of $\hat{\Gamma}$. However, by the celebrated theorem of Nikolov and Segal which asserts that every finite index subgroup of a finitely generated profinite group is open [NS, whenever $\Gamma$ is finitely generated, the group of continuous automorphisms of $\hat{\Gamma}$ is equal to the group of automorphisms of $\hat{\Gamma}$.
} 
$\Gamma$ be a finitely generated group, $\bar{\Gamma}=\Gamma /[\Gamma, \Gamma]$ and $\Gamma^{*}=\bar{\Gamma} / \operatorname{tor}(\bar{\Gamma})$, so $\Gamma^{*} \cong \mathbb{Z}^{(d)}$ for some $d$. Denote

$$
\operatorname{Aut}^{*}(\Gamma)=\operatorname{Im}\left(\operatorname{Aut}(\Gamma) \rightarrow \operatorname{Aut}\left(\Gamma^{*}\right)\right) \leq G L_{d}(\mathbb{Z}) .
$$

When $\Gamma$ is nilpotent, the group $A u t^{*}(\Gamma)$ is known to be an arithmetic subgroup of $G L_{d}(\mathbb{Z})$, and every arithmetic subgroup of $G L_{d}(\mathbb{Z})$ is obtained like that for some nilpotent group $\Gamma([\mathrm{Ba}, \overline{\mathrm{BG}}, \overline{\mathrm{BP}})$.

The canonical map $\operatorname{Aut}(\Gamma) \rightarrow A u t^{*}(\Gamma)$ induces a map

$$
C(\Gamma) \rightarrow C\left(A u t^{*}(\Gamma), \Gamma^{*}\right) .
$$

Here is the main theorem of the paper:

Theorem 1.1. Let $\Gamma$ be a finitely generated nilpotent group. Then, the canonical map $C(\Gamma) \rightarrow C\left(A u t^{*}(\Gamma), \Gamma^{*}\right)$ is an isomorphism.

So, the CSP for nilpotent groups is completely reduced to the classic CSP. In particular, in the free cases, we have:

Corollary 1.2. Let $\Gamma=\Psi_{n, c}$ be the free nilpotent group of class $c$ on $n$ elements. Then

$$
C(\Gamma) \cong C\left(A u t^{*}(\Gamma), \Gamma^{*}\right)=C\left(G L_{n}(\mathbb{Z}), \mathbb{Z}^{(n)}\right)=C\left(\mathbb{Z}^{(n)}\right) .
$$

In particular:

- For $n=2$ one has $C\left(\Psi_{2, c}\right) \cong C\left(\mathbb{Z}^{(2)}\right) \cong \hat{F}_{\omega}$.

- For $n \geq 3$ one has $C\left(\Psi_{n, c}\right) \cong C\left(\mathbb{Z}^{(n)}\right) \cong\{e\}$.

Remark 1.3. As mentioned above, every arithmetic subgroup $D$ of $G L_{d}(\mathbb{Z})$ can appear as $\operatorname{Aut}^{*}(\Gamma)$ for a suitable nilpotent $\Gamma$. The possible congruence kernels for such arithmetic groups are not fully known as the classical CSP is not yet fully solved. But these include, besides the trivial groups and $\hat{F}_{\omega}$ mentioned above, also finite cyclic groups (when $D$ is the restriction of scalars from suitable number fields) as well as infinite abelian groups of finite exponent (if $D$ is an arithmetic group of a non simply connected group).

Here is the main line of the proof. For a finitely generated group $\Gamma$ consider the commutative exact diagram

$$
\begin{aligned}
& 1 \rightarrow A^{*}(\Gamma) \rightarrow \operatorname{Aut}(\Gamma) \rightarrow \operatorname{Aut}^{*}(\Gamma) \rightarrow 1 \\
& 1 \rightarrow I A^{*}(\hat{\Gamma}) \rightarrow \underset{\text { Aut }}{\downarrow}(\hat{\Gamma}) \rightarrow \text { Aut }^{*}(\hat{\Gamma}) \rightarrow 1
\end{aligned}
$$

when we define $I A^{*}(\Gamma)=\operatorname{ker}\left(A u t(\Gamma) \rightarrow \operatorname{Aut}\left(\Gamma^{*}\right)\right)$ and $A u t^{*}(\hat{\Gamma}), I A^{*}(\hat{\Gamma})$ defined to be the image and the kernel of the natural map $A u t(\hat{\Gamma}) \rightarrow A u t\left(\widehat{\Gamma^{*}}\right)=G L_{d}(\hat{\mathbb{Z}})$, respectively. This diagram gives rise to the commutative exact diagram (see Lemma 2.1 in [BER]

$$
\begin{aligned}
& \widehat{I A^{*}(\Gamma)} \rightarrow \widehat{\operatorname{Aut}(\Gamma)} \rightarrow \widehat{A u t^{*}(\Gamma)} \rightarrow 1
\end{aligned}
$$

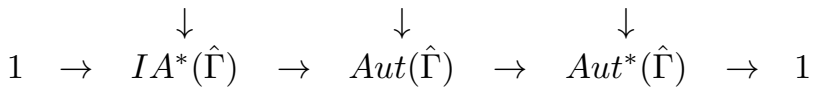


Notice that $\left.C\left(A u t^{*}(\Gamma), \Gamma^{*}\right)=\operatorname{ker}\left(\widehat{A u t^{*}(\Gamma}\right) \rightarrow A u t^{*}(\hat{\Gamma})\right)$. We prove the following theorem:

Theorem 1.4. Let $\Gamma$ be a finitely generated nilpotent group. Then:

1. For any $G \leq I A^{*}(\Gamma)$, the natural map $G \rightarrow I A^{*}(\hat{\Gamma})$ is an embedding. In other words

$$
C(G, \Gamma)=\{e\}
$$

so we have an affirmative solution to the $C S P$ for any $G \leq I A^{*}(\Gamma)$.

2. The group $I A^{*}(\Gamma)$ is dense in $I A^{*}(\hat{\Gamma})$.

Notice that from the first part of Theorem 1.4 we obtain that in particular $C\left(I A^{*}(\Gamma), \Gamma\right)=\{e\}$ for any finitely generated nilpotent group $\Gamma$. This is not true in general (cf. $\mathrm{Be} 1, \mathrm{Be} 2, \mathrm{Be} 3, \mathrm{BL}$ for free metabelian groups). In some sense, the second part of Theorem 1.4 means that the map $I A^{*}(\Gamma) \rightarrow I A^{*}(\hat{\Gamma})$ satisfies a "strong approximation" propery. This is not true in general either (compare [L2] for free groups). From the two parts of Theorem [1.4 we obtain the following corollary, which also implies Theorem 1.1:

Corollary 1.5. Let $\Gamma$ be a finitely generated nilpotent group. Then

$$
\widehat{I A^{*}(\Gamma)} \cong I A^{*}(\hat{\Gamma})
$$

Corollary 1.5, together with chasing diagram (1.1), imply Theorem 1.1 Corollary 1.5 is a form of combination of congruence subgroup property as well as strong approximation for the group $I A^{*}(\Gamma)$. Indeed, its proof boils down to these results for a suitable $\mathbb{Q}$-unipotent group. But the reduction is slightly delicate: in 93 , it is shown that the proof of Corollary 1.5 (or Theorem 1.4) can be reduced to the case when $\Gamma$ is torsion free. In $\$ 2$, we treat the torsion free case, by reducing it first from $\Gamma$ to $\Delta$, when $\Delta$ is the "lattice hull" of $\Gamma$. This $\Delta$ contains $\Gamma$ as a finite index subgroup and it is contained in its Mal'cev completion $R$. It enjoys the property that $\log (\Delta)$ is a $\mathbb{Z}$-lattice of the Lie algebra $L$ of $R$. This fact enables us to give $I A^{*}(\Delta)$ the structure of the $\mathbb{Z}$-points of a suitable unipotent group for which the $\hat{\mathbb{Z}}$-points are exactly $I A^{*}(\hat{\Delta})$. Hence, the classical CSP and strong approximation for this unipotent group imply Corollary 1.5 .

In 4 we sketch another proof to Corollary 1.5, which is more direct, in the case where $\Gamma=\Psi_{n, c}$ is a finitely generated free nilpotent group.

Acknowledgments: During the period of the research, the first author was supported by the Rudin foundation and, not concurrently, by NSF research training grant (RTG) \# 1502651. The second author is indebted for support from the NSF (Grant No. DMS-1700165) and the European Research Council (ERC) under the European Unions Horizon 2020 research and innovation program (Grant No. 692854). 


\section{The case of Torsion Free Nilpotent Groups}

In this section we are going to prove Theorem 1.4 in the case where $\Gamma$ is torsion free. So let $\Gamma$ be a finitely generated torsion free nilpotent group. For our convenience, we will follow the approach presented in $[\underline{S}$, and consider $\Gamma$ as a subgroup of $\operatorname{Tr}_{1}(n, \mathbb{Z})$, the group of $n \times n$ upper triangular matrices over $\mathbb{Z}$ with 1 -s on the diagonal, for some $n$ (see Chapter 5 therein). Recall the one-to-one correspondence given by the maps

$$
\begin{aligned}
\log : \operatorname{Tr}_{1}(n, \mathbb{Q}) & \rightarrow \operatorname{Tr}_{0}(n, \mathbb{Q}) \\
\exp : \operatorname{Tr}_{0}(n, \mathbb{Q}) & \rightarrow \operatorname{Tr}_{1}(n, \mathbb{Q})
\end{aligned}
$$

where $\operatorname{Tr}_{0}(n, \mathbb{Q})$ is the Lie algebra of $n \times n$ upper triangular matrices with 0 -s on the diagonal. Let $L$ be the Lie subalgebrs of $\operatorname{Tr}_{0}(n, \mathbb{Q})$ spanned by $\log (\Gamma)$. The following is well known and can be found in [S] as well (Chapter 6):

Theorem 2.1. There exists a unique (up to isomorphism) group $R$, called the radicable hull of $\Gamma$, or Mal'cev completion of $\Gamma$ with the following properties:

- $\Gamma$ is a subgroup of $R$.

- For every $a \in R$ and $m \in \mathbb{N}$ there exists $b \in R$ such that $b^{m}=a$.

- For every $a \in R$ there exists $m \in \mathbb{N}$ such that $a^{m} \in \Gamma$.

- The group $R$ can be identified with $\exp (L) \leq \operatorname{Tr}_{1}(n, \mathbb{Q})$.

The connection between the group operation of $R$ and the Lie algebra opration of $L$ is given through the Baker-Campbell-Hausdorf (BCH) formula. One can use it in order to prove the following lemma ([BG], Lemma 2.1):

Lemma 2.2. Under the correspondence between the underlying sets of $R$ and $L$ one has $R^{\prime}=L^{\prime}$. This equality gives a natural group isomorphism between $R / R^{\prime}$ and the additive group $L / L^{\prime}$.

One can use the BCH formula in order to prove that $L^{\prime}$ is the $\mathbb{Q}$-span of $\log \left(\Gamma^{\prime}\right)$, and hence $R^{\prime}$ can be identified with the radicable hull of $\Gamma^{\prime}$. Denote $\delta(\Gamma)=\operatorname{ker}\left(\Gamma \rightarrow \Gamma^{*} \simeq \mathbb{Z}^{d}\right)$. Then, as any element of $\delta(\Gamma)$ has some power in $\Gamma^{\prime}$, we have $\log (\delta(\Gamma)) \subseteq L^{\prime}$, and hence $L^{\prime}$ is also the $\mathbb{Q}$-span of $\log (\delta(\Gamma))$, and $R^{\prime}$ is also the radicable hull of $\delta(\Gamma)$. One gets from this that ([BG], Lemma 2.2):

Lemma 2.3. We have $\Gamma \cap R^{\prime}=\delta(\Gamma)$ and $\operatorname{dim}_{\mathbb{Q}}\left(R / R^{\prime}\right)=\operatorname{rank}_{\mathbb{Z}}(\Gamma / \delta(\Gamma))=d$.

The following can be found in $[\underline{S}$, Chapter 6 :

Proposition 2.4. There exists a unique minimal intermediate subgroup $\Gamma \leq$ $\Delta \leq R$, called the lattice hull of $\Gamma$, that its image in $L$, namely $\log (\Delta)$, is a lattice. I.e. $\log (\Delta) \overline{\text { is a free } \mathbb{Z}}$-module that spans $L$ over $\mathbb{Q}$. One has $[\Delta: \Gamma]<$ $\infty$.

Remark 2.5. Notice that $R$ is also the radicable hull of $\Delta$. 
Given a set $X \subseteq L$ denote $N_{A u t(L)}(X)=\{g \in \operatorname{Aut}(L) \mid g(X)=X\}$. The following can also be found in [S], Chapter 6 :

Theorem 2.6. The correspondence between $R$ and $L$ induces an isomorphism

$$
\operatorname{Aut}(R) \simeq \operatorname{Aut}(L) \leq G L_{k}(\mathbb{Q})
$$

where $k=\operatorname{dim}(L)$. Under this isomorphism, one can identify

$$
\begin{aligned}
A u t(\Gamma) \cong N_{\text {Aut }(L)}(\log (\Gamma)) \leq \operatorname{Aut}(L) \\
\operatorname{Aut}(\Delta) \cong N_{\text {Aut }(L)}(\log (\Delta)) \leq \operatorname{Aut}(L) .
\end{aligned}
$$

From Proposition 2.4, it follows that $\operatorname{Aut}(\Gamma) \leq \operatorname{Aut}(\Delta) \leq \operatorname{Aut}(R) \simeq \operatorname{Aut}(L)$. I.e. any automorphism of $\Gamma$ can be uniquely extended to an automorphism of $\Delta$, and any of the latter can be uniquely extended to an automorphism of $R$.

Theorem 2.6 and Lemma 2.3 imply:

Proposition 2.7. Under the above notation we can identify

$$
\begin{aligned}
I A^{*}(\Gamma) & =\operatorname{Aut}(\Gamma) \cap \operatorname{ker}\left(\operatorname{Aut}(R) \rightarrow \operatorname{Aut}\left(R / R^{\prime}\right)\right) \\
& \cong N_{\text {Aut }(L)}(\log (\Gamma)) \cap \operatorname{ker}\left(\operatorname{Aut}(L) \rightarrow \operatorname{Aut}\left(L / L^{\prime}\right)\right) \\
I A^{*}(\Delta) & =\operatorname{Aut}(\Delta) \cap \operatorname{ker}\left(\operatorname{Aut}(R) \rightarrow \operatorname{Aut}\left(R / R^{\prime}\right)\right) \\
& \cong N_{\text {Aut }(L)}(\log (\Delta)) \cap \operatorname{ker}\left(\operatorname{Aut}(L) \rightarrow \operatorname{Aut}\left(L / L^{\prime}\right)\right) .
\end{aligned}
$$

An immediate corollary of Proposition 2.7 and Theorem 2.6 is that $I A^{*}(\Gamma)$ is naturally embedded in $I A^{*}(\Delta)$. We would like now to show that the same property is valid also for the profinte completions of $\Gamma$ and $\Delta$. Namely:

Proposition 2.8. Any automorphism of $\hat{\Gamma}$ can be uniquely extended to an automorphism of $\hat{\Delta}$. In particular, $I A^{*}(\hat{\Gamma})$ is naturally embedded as a finite index subgroup of $I A^{*}(\hat{\Delta})$.

In order to prove Proposition 2.8, we are going to show that one can describe the relation between $I A^{*}(\hat{\Gamma})$ and $I A^{*}(\hat{\Delta})$ in a very similar way to the description of the relation between $I A^{*}(\Gamma)$ and $I A^{*}(\Delta)$ above. Before we do that, let us present an immediate consequence of Proposition 2.8.

Proposition 2.9. Let $\Gamma$ be a finitely generated torsion free nilpotent group, and let $\Delta$ be the lattice hull of $\Gamma$. Let $G \leq I A^{*}(\Gamma) \leq I A^{*}(\Delta)$. Then:

1. If $\hat{G} \rightarrow I A^{*}(\hat{\Delta})$ is injective, then $\hat{G} \rightarrow I A^{*}(\hat{\Gamma})$ is injective.

2. If $I A^{*}(\Delta)$ is dense in $I A^{*}(\hat{\Delta})$, then $I A^{*}(\Gamma)$ is dense in $I A^{*}(\hat{\Gamma})$.

Proof. The first statment is an immediate corollary of Proposition 2.8 The second part also follows immediately from Proposition 2.8 since $I A^{*}(\Delta) \cap I A^{*}(\hat{\Gamma})=$ $I A^{*}(\Gamma)$. 
Proposition 2.9 shows us that in order to prove Theorem 1.4 for finitely generated torsion free nilpotent group $\Gamma$, it is enough to show it for its lattice hull $\Delta$. We turn now to describe the relation between $\operatorname{Aut}(\hat{\Gamma})$ and $A u t(\hat{\Delta})$. The description is going to give more than just a proof to Proposition 2.8, and we are going to use it also toward the rest of the section.

Let $\Gamma_{p}$ be the pro- $p$ completion of $\Gamma$. As $\Gamma$ is nilpotent, we have $\hat{\Gamma}=\prod_{p} \Gamma_{p}$. In addition, as $\Gamma$ is finitely generated and unipotent, it is arithmetic ([S], Chapter 6). Hence, by the affirmative solution to the congruence subgroup problem for arithmetic soluble groups (see [C, $[\mathrm{P},[\mathrm{PS}]$ ), we can view $\hat{\Gamma}$ as the closure of $\Gamma$ under the map

$$
\Gamma \hookrightarrow \operatorname{Tr}_{1}(n, \mathbb{Z}) \rightarrow \operatorname{Tr}_{1}(n, \hat{\mathbb{Z}})=\prod_{p} \operatorname{Tr}_{1}\left(n, \mathbb{Z}_{p}\right) .
$$

As $\operatorname{Tr}_{1}\left(n, \mathbb{Z}_{p}\right)$ is a pro- $p$ group, and $\hat{\Gamma}=\prod_{p} \Gamma_{p}$, it follows that we can identify $\Gamma_{p}$ with the closure of $\Gamma$ under the map

$$
\Gamma \hookrightarrow \operatorname{Tr}_{1}(n, \mathbb{Z}) \rightarrow \prod_{p} \operatorname{Tr}_{1}\left(n, \mathbb{Z}_{p}\right) \rightarrow \operatorname{Tr}_{1}\left(n, \mathbb{Z}_{p}\right) .
$$

Extending log $: \operatorname{Tr}_{1}\left(n, \mathbb{Q}_{p}\right) \rightarrow \operatorname{Tr}_{0}\left(n, \mathbb{Q}_{p}\right)$ and $\exp : \operatorname{Tr}_{0}\left(n, \mathbb{Q}_{p}\right) \rightarrow \operatorname{Tr}_{1}\left(n, \mathbb{Q}_{p}\right)$, $\log$ and exp are continuous with relation to the topology induced by $\mathbb{Q}_{p}$. We define $L_{p}$ to be the $\mathbb{Q}$-span of $\log \left(\Gamma_{p}\right)$ and $R_{p}=\exp \left(L_{p}\right)$.

Lemma 2.10. The set $L_{p}$ is a $\mathbb{Q}_{p}$-Lie algebra.

Proof. The BCH clearly gives $L_{p}$ a structure of a $\mathbb{Q}$-Lie algebra, just like it gives $L$. We just need to explain why $L_{p}$ is closed under multiplication of scalars from $\mathbb{Q}_{p}$. By definition, it is enough to show that it is closed under multiplication of scalars from $\mathbb{Z}_{p}$. So let $g \in \Gamma_{p}$ and let $m=\lim _{i \rightarrow \infty} m_{i} \in \mathbb{Z}_{p}$ for some $m_{i} \in \mathbb{Z}$. Let $\rho_{g}$ be the natural homomorphism $\rho_{g}: \mathbb{Z}_{p} \rightarrow \stackrel{i \rightarrow \infty}{\Gamma_{p}}$ defined by sending the generator of $\mathbb{Z}_{p}$ to $g$. Then, as log is continuous we have

$$
\begin{aligned}
\log \left(\rho_{g}(m)\right) & =\log \left(\rho_{g}\left(\lim _{i \rightarrow \infty} m_{i}\right)\right)=\lim _{i \rightarrow \infty} \log \left(\rho_{g}\left(m_{i}\right)\right)=\lim _{i \rightarrow \infty} \log \left(g^{m_{i}}\right) \\
& =\lim _{i \rightarrow \infty}\left(m_{i} \cdot \log (g)\right)=\left(\lim _{i \rightarrow \infty} m_{i}\right) \cdot \log (g)=m \cdot \log (g) .
\end{aligned}
$$

It follows that the set $\log \left(\Gamma_{p}\right)$ is closed under multiplication by elements from $\mathbb{Z}_{p}$, and so is $L_{p}$.

The proof of the following is similar to the proof of the corresponding properties of the Mal'cev completion in Theorem 2.1.

Lemma 2.11. The set $R_{p}$ is a group containing $\Gamma, R$ and $\Gamma_{p}$. Moreover, $R_{p}$ is a Mal'cev completion of $\Gamma_{p}$ in the sense that it satisfies the following properties:

- For every $a \in R_{p}$ and $m \in \mathbb{N}$ there exists $b \in R_{p}$ such that $b^{m}=a$.

- For every $a \in R_{p}$ there exists $m \in \mathbb{N}$ such that $a^{m} \in \Gamma_{p}$. 
Also, similarly to Lemma 2.2. one can use the $\mathrm{BCH}$ formula in order to show:

Lemma 2.12. Under the correspondence between $R_{p}$ and $L_{p}$ one has $R_{p}^{\prime}=$ $L_{p}^{\prime}$. This equality gives a natural group homomorphism between $R_{p} / R_{p}^{\prime}$ and the additive group $L_{p} / L_{p}^{\prime}$.

One can use the BCH formula in order to prove that $L_{p}^{\prime}$ is the $\mathbb{Q}$-span of $\log \left(\Gamma_{p}^{\prime}\right)$, and hence $R_{p}^{\prime}$ is a Mal'cev completion of $\Gamma_{p}^{\prime}$ in the sense of Lemma 2.11. Denoting $\delta\left(\Gamma_{p}\right)=\operatorname{ker}\left(\Gamma_{p} \rightarrow\left(\Gamma^{*}\right)_{p} \simeq \mathbb{Z}_{p}^{d}\right)$ where $d=\operatorname{rank}_{\mathbb{Z}}\left(\Gamma^{*}\right)$, we have also a similar property as in Lemma 2.3 .

Lemma 2.13. One has $\Gamma_{p} \cap R_{p}^{\prime}=\delta\left(\Gamma_{p}\right)$ and

$$
\operatorname{dim}_{\mathbb{Q}_{p}}\left(R_{p} / R_{p}^{\prime}\right)=\operatorname{rank}_{\mathbb{Z}_{p}}\left(\Gamma_{p} / \delta\left(\Gamma_{p}\right)\right)=d .
$$

Proof. We first prove that $\delta\left(\Gamma_{p}\right)=\operatorname{ker}\left(\Gamma_{p} \rightarrow \bar{\Gamma}_{p} / \operatorname{tor}\left(\bar{\Gamma}_{p}\right)\right)$, where $\bar{\Gamma}_{p}=\Gamma_{p} / \Gamma_{p}^{\prime}$. As $\left(\Gamma^{*}\right)_{p}$ is a torsion free abelian quotient of $\Gamma_{p}$, it follows that $\delta\left(\Gamma_{p}\right) \supseteq \operatorname{ker}\left(\Gamma_{p} \rightarrow\right.$ $\left.\bar{\Gamma}_{p} / \operatorname{tor}\left(\bar{\Gamma}_{p}\right)\right)$. On the other hand, the map $\Gamma \rightarrow \Gamma_{p}$ induces a map $\Gamma^{*} \rightarrow$ $\bar{\Gamma}_{p} / \operatorname{tor}\left(\bar{\Gamma}_{p}\right)$. Now, as $\Gamma_{p}$ is a finitely generated pro- $p$ group, $\Gamma_{p}^{\prime}$ is closed in $\Gamma_{p}$ (see Proposition 1.19 in [DDSM]). Hence, $\Gamma_{p} \rightarrow \bar{\Gamma}_{p}$ is a continuous homomorphism, and hence, $\Gamma_{p} \rightarrow \bar{\Gamma}_{p} / \operatorname{tor}\left(\bar{\Gamma}_{p}\right)$ is continuous as well. Thus, we can say that the image of $\Gamma^{*}$ under the map $\Gamma^{*} \rightarrow \bar{\Gamma}_{p} / \operatorname{tor}\left(\bar{\Gamma}_{p}\right)$, is dense in $\bar{\Gamma}_{p} / \operatorname{tor}\left(\bar{\Gamma}_{p}\right)$. Hence, we have a surjective continuous homomorphism $\left(\Gamma^{*}\right)_{p} \rightarrow \bar{\Gamma}_{p} / \operatorname{tor}\left(\bar{\Gamma}_{p}\right)$. It follows that $\delta\left(\Gamma_{p}\right) \subseteq \operatorname{ker}\left(\Gamma_{p} \rightarrow \bar{\Gamma}_{p} / \operatorname{tor}\left(\bar{\Gamma}_{p}\right)\right)$, so $\delta\left(\Gamma_{p}\right)=\operatorname{ker}\left(\Gamma_{p} \rightarrow \bar{\Gamma}_{p} / \operatorname{tor}\left(\bar{\Gamma}_{p}\right)\right)$ as required.

Now, as $R_{p}$ contains $\Gamma_{p}$, we have $\Gamma_{p} /\left(\Gamma_{p} \cap R_{p}^{\prime}\right) \leq R_{p} / R_{p}^{\prime}$. It follows that $\Gamma_{p} /\left(\Gamma_{p} \cap R_{p}^{\prime}\right)$ is a torsion free abelian quotient of $\Gamma_{p}$. Hence

$$
\delta\left(\Gamma_{p}\right)=\operatorname{ker}\left(\Gamma_{p} \rightarrow \bar{\Gamma}_{p} / \operatorname{tor}\left(\bar{\Gamma}_{p}\right)\right) \subseteq \Gamma_{p} \cap R_{p}^{\prime} .
$$

On the other hand, as every element of $R_{p}^{\prime}$ has a power in $\Gamma_{p}^{\prime}$, it follows that we also have $\Gamma_{p} \cap R_{p}^{\prime} \subseteq \operatorname{ker}\left(\Gamma_{p} \rightarrow \bar{\Gamma}_{p} / \operatorname{tor}\left(\bar{\Gamma}_{p}\right)\right)=\delta\left(\Gamma_{p}\right)$, as required.

The equality $\Gamma_{p} \cap R_{p}^{\prime}=\delta\left(\Gamma_{p}\right)$ implies that $\Gamma_{p} / \delta\left(\Gamma_{p}\right) \leq R_{p} / R_{p}^{\prime}$. Thus $\Gamma_{p} / \delta\left(\Gamma_{p}\right)$ is a free $\mathbb{Z}_{p}$-module that spanes the vector space $R_{p} / R_{p}^{\prime}$ over $\mathbb{Q}_{p}$. Hence $\operatorname{dim}_{\mathbb{Q}_{p}}\left(R_{p} / R_{p}^{\prime}\right)=\operatorname{rank}_{\mathbb{Z}_{p}}\left(\Gamma_{p} / \delta\left(\Gamma_{p}\right)\right)=d$, as required.

The following corollary will be needed later:

Corollary 2.14. We have $\operatorname{dim}_{\mathbb{Q}}(L)=\operatorname{dim}_{\mathbb{Q}_{p}}\left(L_{p}\right)$.

Proof. We saw that

$$
\begin{aligned}
\operatorname{dim}_{\mathbb{Q}_{p}}\left(L_{p} / L_{p}^{\prime}\right) & =\operatorname{dim}_{\mathbb{Q}_{p}}\left(R_{p} / R_{p}^{\prime}\right)=\operatorname{rank}_{\mathbb{Z}_{p}}\left(\Gamma_{p} / \delta\left(\Gamma_{p}\right)\right)=d \\
& =\operatorname{rank}_{\mathbb{Z}}(\Gamma / \delta(\Gamma))=\operatorname{dim}_{\mathbb{Q}}\left(R / R^{\prime}\right)=\operatorname{dim}_{\mathbb{Q}}\left(L / L^{\prime}\right) .
\end{aligned}
$$

By the fact that the commutator subgroup of a finitely generated niplotent group is finitely generated, and $L^{\prime}$ is the $\mathbb{Q}$-span of $\log \left(\Gamma^{\prime}\right)$ one has

$$
\operatorname{rank}_{\mathbb{Z}_{p}}\left(\left(\Gamma^{\prime}\right)_{p} / \delta\left(\left(\Gamma^{\prime}\right)_{p}\right)\right)=\operatorname{rank}_{\mathbb{Z}}\left(\Gamma^{\prime} / \delta\left(\Gamma^{\prime}\right)\right)=\operatorname{dim}_{\mathbb{Q}}\left(L^{\prime} / L^{\prime \prime}\right) .
$$


Using the CSP for arithmetic soluble groups, we can identify $\left(\Gamma^{\prime}\right)_{p}$ with the closure of $\Gamma^{\prime}$ in $\Gamma_{p} \leq \operatorname{Tr}_{1}\left(n, \mathbb{Z}_{p}\right)$. As explained in the proof of Lemma 2.13, the latter can be identified with $\left(\Gamma_{p}\right)^{\prime}$, and hence $\left(\Gamma^{\prime}\right)_{p}=\left(\Gamma_{p}\right)^{\prime}$. Hence, $L_{p}^{\prime}$, which is the $\mathbb{Q}$-span of $\log \left(\left(\Gamma_{p}\right)^{\prime}\right)$, is actually the $\mathbb{Q}$-span of $\log \left(\left(\Gamma^{\prime}\right)_{p}\right)$. It follows that

$$
\operatorname{dim}_{\mathbb{Q}_{p}}\left(L_{p}^{\prime} / L_{p}^{\prime \prime}\right)=\operatorname{rank}_{\mathbb{Z}_{p}}\left(\left(\Gamma^{\prime}\right)_{p} / \delta\left(\left(\Gamma^{\prime}\right)_{p}\right)\right)=\operatorname{dim}_{\mathbb{Q}}\left(L^{\prime} / L^{\prime \prime}\right) .
$$

Continuing like that, we obtain that $\operatorname{dim}_{\mathbb{Q}}\left(L^{(i)} / L^{(i+1)}\right)=\operatorname{dim}_{\mathbb{Q}_{p}}\left(L_{p}^{(i)} / L_{p}^{(i+1)}\right)$ for any $i$, where $L^{(i)}, L_{p}^{(i)}$ are the $i$-th derivatives of $L, L_{p}$ respectively. Therefore

$$
\operatorname{dim}_{\mathbb{Q}}(L)=\sum_{i} \operatorname{dim}_{\mathbb{Q}}\left(L^{(i)} / L^{(i+1)}\right)=\sum_{i} \operatorname{dim}_{\mathbb{Q}_{p}}\left(L_{p}^{(i)} / L_{p}^{(i+1)}\right)=\operatorname{dim}_{\mathbb{Q}_{p}}\left(L_{p}\right)
$$

as required.

Remark 2.15. We presented a proof for Corollary 2.14, based on the previous line of discussion, but the knowledgeable reader can also deduce it by recalling that $\operatorname{dim}_{\mathbb{Q}}(L)$ is equal to $h(\Gamma)$ - the Hirsch length of $\Gamma$, and $h(\Gamma)$ is equal to $\operatorname{dim}\left(\Gamma_{p}\right)$ - the dimension of the pro- $p$ completion of $\Gamma$, and the later is equal to $\operatorname{dim}_{\mathbb{Q}_{p}}\left(L_{p}\right)$ as $L_{p}$ is the Lie algebra of $\Gamma_{p}$.

Recall $\Delta$, the lattice hull of $\Gamma$. For our convenience, without loss of generality, we can assume that $\Gamma \leq \Delta \leq \operatorname{Tr}_{1}(n, \mathbb{Z})$ (see Lemma 2 in Chapter 6 of $[\mathbf{S}]$ ). Hence, $\Delta_{p}$ can be identified with the closure of $\Delta$ in $\operatorname{Tr}_{1}\left(n, \mathbb{Z}_{p}\right)$.

Lemma 2.16. One has $\log \left(\Delta_{p}\right)=\mathbb{Z}_{p} \log (\Delta) \subseteq L_{p}$, where $\mathbb{Z}_{p} \log (\Delta)$ is the $\mathbb{Z}_{p^{-}}$ lattice of $L_{p}$ sppaned by $\log (\Delta)$. In particular, $\log \left(\Delta_{p}\right)$ is a $\mathbb{Z}_{p}$-lattice in $L_{p}$ and $\Gamma_{p} \leq \Delta_{p} \leq R_{p}$.

Proof. By a similar argument as in Lemma 2.10, for any element $m \in \mathbb{Z}_{p}$ and $a \in \log (\Delta)$ we have $m \cdot a \in \log \left(\Delta_{p}\right)$. Hence, we will get $\log \left(\Delta_{p}\right) \supseteq \mathbb{Z}_{p} \log (\Delta)$ once we show that for any $g, h \in \Delta_{p}$ there exists $k \in \Delta_{p}$ such that $\log (g)+\log (h)=$ $\log (k)$. By assumption, the group $\Delta$ is dense in $\Delta_{p}$ where the topology on $\Delta_{p}$ coincides with the topology induced by $\operatorname{Tr}_{1}\left(\mathbb{Q}_{p}\right)$. Let $g_{i}, h_{i}, k_{i} \in \Delta$ be such that

$$
\lim _{i \rightarrow \infty} g_{i}=g \quad \lim _{i \rightarrow \infty} h_{i}=h \quad \log \left(k_{i}\right)=\log \left(g_{i}\right)+\log \left(h_{i}\right) .
$$

We need to show that $\lim _{i \rightarrow \infty} k_{i}=k \in \Delta_{p}$ exists and $\log (g)+\log (h)=\log (k)$. As exp and log are continuous, we have

$$
\begin{aligned}
k & =\lim _{i \rightarrow \infty} k_{i}=\lim _{i \rightarrow \infty} \exp \left(\log \left(g_{i}\right)+\log \left(h_{i}\right)\right) \\
& =\exp \left(\log \left(\lim _{i \rightarrow \infty} g_{i}\right)+\log \left(\lim _{i \rightarrow \infty} h_{i}\right)\right)=\exp (\log (g)+\log (h)) .
\end{aligned}
$$

As $\Delta_{p}$ is compact, we have $k \in \Delta_{p}$ where $\log (g)+\log (h)=\log (k)$.

For the opposite inclusion: $\Delta$ is dense in $\Delta_{p}$, so $\log (\Delta)$ is dense in $\log \left(\Delta_{p}\right)$. As $\mathbb{Z}_{p} \log (\Delta)$ is closed in $L_{p}$, it follows that $\log \left(\Delta_{p}\right) \subseteq \mathbb{Z}_{p} \log (\Delta)$, as required. 
Lemma 2.17. The group $\Delta_{p}$ is a unique minimal subgroup $\Gamma_{p} \leq \Delta_{p} \leq R_{p}$ that its image in $L_{p}$ is a $\mathbb{Z}_{p}$-lattice.

Proof. Suppose that $\Lambda_{p}$ satisfies $\Gamma_{p} \leq \Lambda_{p} \leq R_{p}, \log \left(\Lambda_{p}\right)$ is a $\mathbb{Z}_{p}$-lattice, and $\log \left(\Delta_{p}\right) \nsubseteq \log \left(\Lambda_{p}\right)$. It follows that $\Gamma \leq \Lambda_{p} \cap \Delta \lesseqgtr \Delta$ where $\log \left(\Lambda_{p} \cap \Delta\right)=$ $\log \left(\Lambda_{p}\right) \cap \log (\Delta)$ is a lattice of $L$ that contains $\log (\Gamma)$. This is a contradiction to the minimality of $\Delta$.

Now, using Lemma 2.11 and the property $\Gamma_{p} \leq \Delta_{p} \leq R_{p}$ (Lemma 2.16) ), and following the same steps for the proof of [S] to Theorem 2.6 we have:

Theorem 2.18. There are natural isomorphisms

$$
\begin{aligned}
& \operatorname{Aut}\left(R_{p}\right) \cong \operatorname{Aut}\left(L_{p}\right) \\
& \operatorname{Aut}\left(\Gamma_{p}\right) \cong N_{A u t\left(L_{p}\right)}\left(\log \left(\Gamma_{p}\right)\right) \leq \operatorname{Aut}\left(L_{p}\right) \\
& \operatorname{Aut}\left(\Delta_{p}\right) \cong N_{A u t\left(L_{p}\right)}\left(\log \left(\Delta_{p}\right)\right) \leq \operatorname{Aut}\left(L_{p}\right) .
\end{aligned}
$$

By Lemmas 2.12, 2.13, and Theorem 2.18, we obtain:

Proposition 2.19. Denote $I A^{*}\left(\Gamma_{p}\right)=\operatorname{ker}\left(A u t\left(\Gamma_{p}\right) \rightarrow \operatorname{Aut}\left(\left(\Gamma^{*}\right)_{p}\right)\right.$. Then

$$
I A^{*}\left(\Gamma_{p}\right) \cong N_{A u t\left(L_{p}\right)}\left(\log \left(\Gamma_{p}\right)\right) \cap \operatorname{ker}\left(\operatorname{Aut}\left(L_{p}\right) \rightarrow \operatorname{Aut}\left(L_{p} / L_{p}^{\prime}\right)\right) .
$$

Similarly, as $\Gamma_{p} \leq \Delta_{p} \leq R_{p}$ (Lemma 2.16), we also have

$$
I A^{*}\left(\Delta_{p}\right) \cong N_{\text {Aut }\left(L_{p}\right)}\left(\log \left(\Delta_{p}\right)\right) \cap \operatorname{ker}\left(\operatorname{Aut}\left(L_{p}\right) \rightarrow \operatorname{Aut}\left(L_{p} / L_{p}^{\prime}\right)\right) .
$$

We can now deduce Proposition 2.8.

Proof. (of Proposition 2.8) From Lemma 2.17 and Proposition 2.19 we get that for any prime $p$ one has $I A^{*}\left(\Gamma_{p}\right) \hookrightarrow I A^{*}\left(\Delta_{p}\right)$. Therefore

$$
I A^{*}(\hat{\Gamma})=\prod_{p} I A^{*}\left(\Gamma_{p}\right) \hookrightarrow \prod_{p} I A^{*}\left(\Delta_{p}\right)=I A^{*}(\hat{\Delta}) .
$$

Moreover, $I A^{*}(\hat{\Gamma})=\left\{\alpha \in I A^{*}(\hat{\Delta}) \mid \alpha(\hat{\Gamma})=\hat{\Gamma}\right\}$, hence it is open in $I A^{*}(\hat{\Delta})$.

As we mentioned previously, by Proposition 2.9, in order to prove Theorem 1.4 for $\Gamma$, it is enough to prove it for $\Delta$. So from now on $\log (\Delta)$ is a lattice that spans $L$, and hence $k=\operatorname{dim}_{\mathbb{Q}}(L)=\operatorname{rank}_{\mathbb{Z}}(\log (\Delta))$. Our objective now is to construct a basis for $\log (\Delta)$ that with relation to it, we will be able to view $I A^{*}(\Delta)$ as the $\mathbb{Z}$-points of a $\mathbb{Q}$-algebraic group whose $\mathbb{Z}_{p}$-points are $I A^{*}\left(\Delta_{p}\right)$. Once we show this, we will see that Theorem 1.4 (1) follows from the classical CSP for the arithmetic group $I A^{*}(\Delta)$, and Theorem 1.4 (2) is the classical strong-approximation theorem for this group.

Let $g_{1}, \ldots, g_{d} \in \Delta$ be such that $\Delta^{*}=\Delta / \delta(\Delta) \simeq \mathbb{Z}^{d}$ is generated by the images $\bar{g}_{1}, \ldots, \bar{g}_{d}$. Then, $\bar{g}_{1}, \ldots, \bar{g}_{d}$ also generate $\left(\Delta^{*}\right)_{p}=\Delta_{p} / \delta\left(\Delta_{p}\right) \simeq \mathbb{Z}_{p}^{d}$ as a pro- $p$ group. Hence, by Lemmas 2.3 and 2.13, $l_{1}=\log \left(g_{1}\right), \ldots, l_{d}=\log \left(g_{d}\right)$ generate $R / R^{\prime} \cong L / L^{\prime} \cong \mathbb{Q}^{(d)}$ over $\mathbb{Q}$ and generate $R_{p} / R_{p}^{\prime} \cong L_{p} / L_{p}^{\prime} \cong \mathbb{Q}_{p}^{(d)}$ over $\mathbb{Q}_{p}$. It follows that $l_{1}, \ldots, l_{d}$ are linearly independent over $\mathbb{Q}_{p}$ (and over $\mathbb{Q}$ ) and since $L$ and $L_{p}$ are nilpotent, they generate $L$ as a Lie algebra over $\mathbb{Q}$ and generate $L_{p}$ as a Lie algebra over $\mathbb{Q}_{p}$. 
Lemma 2.20. Let $L=\gamma_{1}(L), L^{\prime}=\gamma_{2}(L), \ldots, 0=\gamma_{c}(L)$ be the lower central series of $L$. The set $l_{1}, \ldots, l_{d}$ can be completed to a basis

$$
B=\left\{l_{1}, \ldots, l_{d}, l_{d+1}, \ldots, l_{k}\right\}
$$

of the lattice $\log (\Delta)$ such that:

1. B contains bases for $\gamma_{j}(L) / \gamma_{j+1}(L) \bmod \gamma_{j+1}(L)$ for every $j$.

2. $l_{j+1}$ lies in the same term as $l_{j}$ in the lower central series of $L$, or deeper.

Proof. Now, $l_{1}, \ldots, l_{d}$ lie in $\gamma_{1}(L)-\gamma_{2}(L)$ and provide a basis for $L / L^{\prime}=\gamma_{1}(L) / \gamma_{2}(L)$ $\bmod \gamma_{2}(L)$. Denote $i_{1}=d$.

For every $j \geq 2$ the group $\left(\log (\Delta) \cap \gamma_{j}(L)\right) /\left(\log (\Delta) \cap \gamma_{j+1}(L)\right)$ is a lattice in $\left.\gamma_{j}(L)\right) / \gamma_{j+1}(L)$ and hence

$$
\left.\operatorname{rank}_{\mathbb{Z}}\left(\left(\log (\Delta) \cap \gamma_{j}(L)\right) /\left(\log (\Delta) \cap \gamma_{j+1}(L)\right)\right)=\operatorname{dim}_{\mathbb{Q}}\left(\gamma_{j}(L)\right) / \gamma_{j+1}(L)\right) .
$$

Let $l_{i_{j-1}+1}, \ldots, l_{i_{j}} \in \log (\Delta) \cap \gamma_{j}(L)$ be a basis to

$$
\left.\left(\log (\Delta) \cap \gamma_{j}(L)\right) /\left(\log (\Delta) \cap \gamma_{j+1}(L)\right) \bmod \log (\Delta) \cap \gamma_{j+1}(L)\right) .
$$

Then $l_{i_{j-1}+1}, \ldots, l_{i_{j}}$ gives a basis for $\left.\gamma_{j}(L)\right) / \gamma_{j+1}(L) \bmod \gamma_{j+1}(L)$ and they all lie in $\gamma_{j}(L)-\gamma_{j+1}(L)$.

This procedure gives us $l_{1}, \ldots, l_{i_{c-1}}$ that satisfy the two conditions in the lemma. Also, by the construction, $l_{1}, \ldots, l_{i_{c-1}}$ span the abelain group $\log (\Delta)$ and provide a basis for $L$. It follows that $i_{c-1}=k$ and that $l_{1}, \ldots, l_{k}$ is the desired basis for $\log (\Delta)$.

Let $B$ be a basis for $\log (\Delta)$ as in the above lemma. Cleary, $B$ is also a basis for $L$ as a vector space over $\mathbb{Q}$. As $L_{p}$ is generated as a Lie algebra by $l_{1}, \ldots, l_{d}$ over $\mathbb{Q}_{p}$, the set $B$ also spans $L_{p}$ over $\mathbb{Q}_{p}$. As $\operatorname{dim}_{\mathbb{Q}}(L)=\operatorname{dim}_{\mathbb{Q}_{p}}\left(L_{p}\right)$ (Proposition 2.14), it follows that $B$ is also a basis for $L_{p}$ as a vector space over $\mathbb{Q}_{p}$. Hence, using the basis $B$ we can identify

$$
\operatorname{Aut}(L)=G L_{k}(\mathbb{Q}) \cap \operatorname{Aut}\left(L_{p}\right) \leq G L_{k}\left(\mathbb{Q}_{p}\right) .
$$

In addition, as $\log (\Delta)$ is a lattice and $\log \left(\Delta_{p}\right)=\mathbb{Z}_{p} \log (\Delta)$ (Lemma 2.16), using the basis $B$ we can identify

$$
\begin{gathered}
\operatorname{Aut}(\Delta)=N_{\text {Aut }(L)}(\log (\Delta))=G L_{k}(\mathbb{Z}) \cap \operatorname{Aut}(L) \\
\operatorname{Aut}\left(\Delta_{p}\right)=N_{\text {Aut }\left(L_{p}\right)}\left(\log \left(\Delta_{p}\right)\right)=G L_{k}\left(\mathbb{Z}_{p}\right) \cap \operatorname{Aut}\left(L_{p}\right) .
\end{gathered}
$$

Moreover, we can identify $\operatorname{Aut}(L)$ and $\operatorname{Aut}\left(L_{p}\right)$ with the groups

$$
\begin{aligned}
\operatorname{Aut}(L) & =\left\{A \in G L_{k}(\mathbb{Q}) \mid[A x, A y]-A[x, y]=0 \quad \forall x, y \in B\right\} \\
\operatorname{Aut}\left(L_{p}\right) & =\left\{A \in G L_{k}\left(\mathbb{Q}_{p}\right) \mid[A x, A y]-A[x, y]=0 \quad \forall x, y \in B\right\}
\end{aligned}
$$

Now, notice that $\sigma \in \operatorname{ker}\left(\operatorname{Aut}(L) \rightarrow \operatorname{Aut}\left(L / L^{\prime}\right)\right)$ if and only if for every $i=1, \ldots, d$ we have $\sigma\left(l_{i}\right) \in l_{i}+L^{\prime}$. As $l_{1}, \ldots, l_{d}$ generate $L$ over $\mathbb{Q}$, it follows that 
for every $\sigma \in \operatorname{ker}\left(\operatorname{Aut}(L) \rightarrow \operatorname{Aut}\left(L / L^{\prime}\right)\right.$ ) and every $i$ (not only for $i=1, \ldots, d$ ) we have

$$
\sigma\left(l_{i}\right)=l_{i}+n_{i}
$$

where $n_{i}$ lies in a strickly deeper term in the lower central series of $L$, than of the one that $l_{i}$ lies in. Hence, by the construction in Lemma 2.20, with relation to $B$ we have

$$
\operatorname{ker}\left(\operatorname{Aut}(L) \rightarrow \operatorname{Aut}\left(L / L^{\prime}\right)\right)=\operatorname{Aut}(L) \cap \operatorname{Tr}_{1}(k, \mathbb{Q}) \leq G L_{k}\left(\mathbb{Q}_{p}\right) .
$$

It follows that $I A^{*}(\Delta)$ can be identified with the arithmetic group of $\mathbb{Z}$-points of $\operatorname{Aut}(L) \cap \operatorname{Tr}_{1}(k, \mathbb{Q})$. Similarly, we have

$$
\operatorname{ker}\left(\operatorname{Aut}\left(L_{p}\right) \rightarrow \operatorname{Aut}\left(L_{p} / L_{p}^{\prime}\right)\right)=\operatorname{Aut}\left(L_{p}\right) \cap \operatorname{Tr}_{1}\left(k, \mathbb{Q}_{p}\right) \leq G L_{k}\left(\mathbb{Q}_{p}\right) .
$$

and hence $I A^{*}\left(\Delta_{p}\right)$ can be identifiead with the $\mathbb{Z}_{p}$-points of $A u t\left(L_{p}\right) \cap \operatorname{Tr}_{1}\left(k, \mathbb{Q}_{p}\right)$. By the description above, $\operatorname{Aut}(L) \cap \operatorname{Tr}_{1}(k, \mathbb{Q})$ and $\operatorname{Aut}\left(L_{p}\right) \cap \operatorname{Tr}_{1}\left(k, \mathbb{Q}_{p}\right)$ are unipotent algebraic groups which are defined by the same equations, over $\mathbb{Q}$ and $\mathbb{Q}_{p}$ respectively.

Now, $I A^{*}(\Delta)$ is a unipotent subgroup of $G L_{k}(\mathbb{Z})$. Hence, by the congruence subgroup property for unipotent arithmetic groups we have

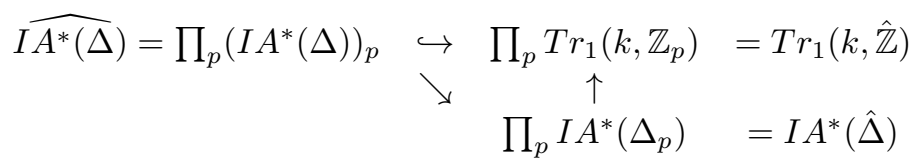

and hence $\widehat{I A^{*}(\Delta)} \hookrightarrow I A^{*}(\hat{\Delta})$ is injective. As $I A^{*}(\Delta)$ is a finitely generated nilpotent group, for any $G \leq I A^{*}(\Delta)$ we have $\hat { G } \hookrightarrow \longdiv { I A ^ { * } ( \Delta ) }$, and hence $\hat{G} \hookrightarrow$ $I A^{*}(\hat{\Delta})$. This gives the first part of Theorem[1.4 in the case of finitely generated torsion free nilpotent groups.

For the second statement of Theorem 1.4 notice that by the above description for $I A^{*}(\Delta)$ and $I A^{*}\left(\Delta_{p}\right)$, we obtain that $I A^{*}(\Delta)$ is dense in $I A^{*}\left(\Delta_{p}\right)$ by the strong approximation property for arithmetic unipotent groups (see [PR], Proposition 7.1, and the corollary afterward). Therefore, as each of the groups $I A^{*}\left(\Delta_{p}\right)$ is a pro- $p$ group, we obtain that $I A^{*}(\Delta)$ is dense in

$$
\prod_{p} I A^{*}\left(\Delta_{p}\right)=I A^{*}(\hat{\Delta}) .
$$

This completes the proof of Theorem 1.4 for finitely generated torsion free nilpotent groups.

\section{The general case}

The aim of this section is to prove Theorem [1.4 given its validity for finitely generated torsion free nilpotent group. Along the section, $\Gamma$ is a finitely generated nilpotent group, and $\Delta=\Gamma / \operatorname{tor}(\Gamma)$. As a finitely generated nilpotent 
group, $\Gamma$ is residually finite. Hence, we can think on $\Gamma$ as a subgroup of $\hat{\Gamma}$. One has $\operatorname{tor}(\hat{\Gamma})=\operatorname{tor}(\Gamma)$ (see Lemma 2.3 in $[\mathrm{KW}$, Corollary 7.5 in $[\mathrm{P}]$ ). In other words, $\operatorname{tor}(\Gamma)$ is a normal subgroup of $\hat{\Gamma}$, and

$$
\hat{\Delta}=\Gamma \widehat{\operatorname{tor}(\Gamma)}=\hat{\Gamma} / \operatorname{tor}(\Gamma)=\hat{\Gamma} / \operatorname{tor}(\hat{\Gamma})
$$

is torsion free. In addition, we get that $\operatorname{tor}(\Gamma)$ is characteristic, not only as a subgroup of $\Gamma$, but also as a subgroup of $\Gamma$. Therefore, we have a map $I A^{*}(\hat{\Gamma}) \rightarrow I A^{*}(\hat{\Delta})$ and we obtain the commutative diagram

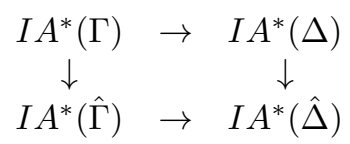

Denote $\tilde{K}=\operatorname{ker}\left(I A^{*}(\Gamma) \rightarrow I A^{*}(\Delta)\right)$. Let $x_{1}, \ldots, x_{n}$ be a generating set for $\Gamma$. Then, every $\alpha \in \tilde{K}$ can be described by

$$
x_{i} \mapsto x_{i} a_{i}
$$

for some $a_{i} \in \operatorname{tor}(\Gamma)$. As $\operatorname{tor}(\Gamma)$ is finite, $\tilde{K}$ is also finite.

Now, let $G \leq I A^{*}(\Gamma)$, and denote its image in $I A^{*}(\Delta)$ by $H$. Then $K=$ $\operatorname{ker}(G \rightarrow H) \leq \tilde{K}$ is finite, and hence we obtain the following commutative exact diagram

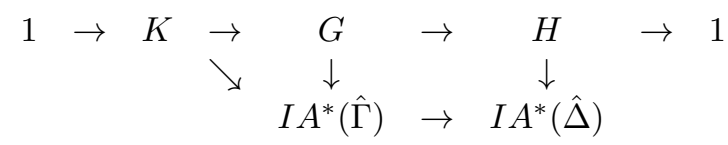

Notice that as $G \leq I A^{*}(\Gamma) \hookrightarrow I A^{*}(\hat{\Gamma})$, the map $K \rightarrow I A^{*}(\hat{\Gamma})$ is injective. Notice also that as $K$ is finite, we have $K=\hat{K}$. Hence, moving to the profinite completion of the upper row, we get the commutative exact diagram

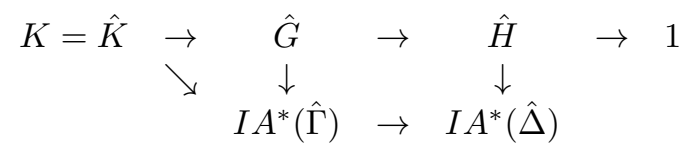

It follows that $\hat{K} \rightarrow I A^{*}(\hat{\Gamma})$ is injective and by $₫ 2$, also $\hat{H} \rightarrow I A^{*}(\hat{\Delta})$ is injective. Hence, by diagram chasing, $\hat{G} \rightarrow I A^{*}(\hat{\Gamma})$ is also injective. This proves the first assertion of Theorem 1.4 in the general case. We move now to prove the second assertion.

As $\Gamma$ is residually finite, and $\operatorname{tor}(\Gamma)$ is finite, there exists $t \in \mathbb{N}$ such that $\operatorname{tor}(\Gamma) \cap \Gamma^{t}=\{e\}$ where $\Gamma^{t}$ is the normal subgroup

$$
\Gamma^{t}=\left\langle g^{t} \mid g \in G\right\rangle \triangleleft \Gamma .
$$

Fix this $t$. It follows that whenever $t \mid m$, we have $\operatorname{tor}(\Gamma) \cap \Gamma^{m}=\{e\}$, and therefore $\operatorname{tor}(\Gamma)$ is naturally embedded in $\Gamma / \Gamma^{m}$ for such $m$. As $\Gamma$ is nilpotent 
and finitely generated, $\Gamma / \Gamma^{m}$ and $\Delta / \Delta^{m}$ are finite for any $m$ (see Corollary 3.3 in $[\mathrm{P}]$ ), and hence one has

$$
\begin{aligned}
& \hat{\Gamma}=\lim _{m \in \mathbb{N}} \Gamma / \Gamma^{m}=\varliminf_{t \mid m} \Gamma / \Gamma^{m} \\
& \hat{\Delta}=\varliminf_{m \in \mathbb{N}} \Delta / \Delta^{m}=\varliminf_{t \mid m} \lim _{m} \Delta / \Delta^{m} .
\end{aligned}
$$

Now, let $\hat{\alpha} \in I A^{*}(\hat{\Gamma})$, and write

$$
\hat{\alpha}=\left(\alpha_{m}\right)_{t \mid m} \in I A^{*}(\hat{\Gamma}) \leq \varliminf_{t \mid m} A u t\left(\Gamma / \Gamma^{m}\right)
$$

where $\alpha_{m} \in \operatorname{Aut}\left(\Gamma / \Gamma^{m}\right)$. In order to prove the second part of Theorem 1.4. namely that $I A^{*}(\Gamma)$ is dense in $I A^{*}(\hat{\Gamma})$, it is enough to show that:

Proposition 3.1. Let $m$ such that $t \mid m$, and let $\hat{\alpha}, \alpha_{m}$ be as above. Then, there exists $\alpha \in I A^{*}(\Gamma)$ such that $\alpha \rightarrow \alpha_{m}$ through the map $I A^{*}(\Gamma) \rightarrow A u t\left(\Gamma / \Gamma^{m}\right)$.

So fix $m$ such that $t \mid m$, and let $\hat{\beta} \in I A^{*}(\hat{\Delta})$ be the image of $\hat{\alpha}$ under the $\operatorname{map} I A^{*}(\hat{\Gamma}) \rightarrow I A^{*}(\hat{\Delta})$. Write

$$
\hat{\beta}=\left(\beta_{m}\right)_{t \mid m} \in I A^{*}(\hat{\Delta}) \leq \lim _{t \mid m} A u t\left(\Delta / \Delta^{m}\right)
$$

where $\beta_{m} \in \operatorname{Aut}\left(\Delta / \Delta^{m}\right)$ is the image of $\alpha_{m} \in \operatorname{Aut}\left(\Gamma / \Gamma^{m}\right)$ under the map $\operatorname{Aut}\left(\Gamma / \Gamma^{m}\right) \rightarrow \operatorname{Aut}\left(\Delta / \Delta^{m}\right)$. By assumption, $I A^{*}(\Delta)$ is dense in $I A^{*}(\hat{\Delta})$, and hence there exists $\beta \in I A^{*}(\Delta)$ such that $\beta \rightarrow \beta_{m}$. So we have the diagram

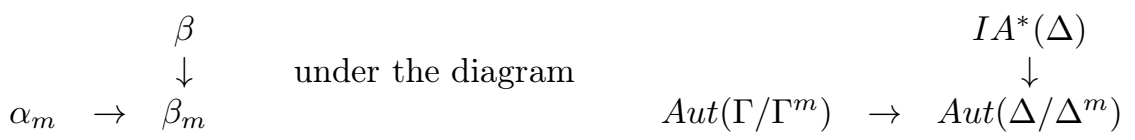

We want to use $\alpha_{m}$ and $\beta$ in order to construct $\alpha \in I A^{*}(\Gamma)$ such that $\alpha \rightarrow \alpha_{m}$.

Let us recall the following notion: let $P_{1}, P_{2}$ and $Q$ be groups with epimorphisms $\pi_{i}: P_{i} \rightarrow Q, i=1,2$. The group

$$
U=P_{1} \times{ }_{Q} P_{2}=\left\{(x, y) \in P_{1} \times P_{2} \mid \pi_{1}(x)=\pi_{2}(y)\right\}
$$

is called the fiber product of $P_{1}, P_{2}$ along $Q$ (or the pullback). It is easy to check that as $\pi_{i}$ are surjective, we get the following commutative diagran of surjective maps

$$
\begin{array}{ccc}
U & \rightarrow & P_{1} \\
\downarrow & & \downarrow \\
P_{2} & \rightarrow & Q
\end{array}
$$

such that $P_{1} \simeq U /\left(U \cap\left(\{e\} \times P_{2}\right)\right)$ and $P_{2} \simeq U /\left(U \cap\left(P_{1} \times\{e\}\right)\right)$. Regarding our context, as we have the commutative surjective diagram

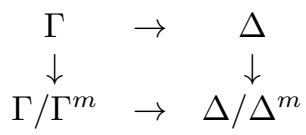


we get a natural map $\Gamma \stackrel{\rho}{\rightarrow} \Delta \times_{\Delta / \Delta^{m}} \Gamma / \Gamma^{m}$. Now, as we assume that $t \mid m$, we have

$$
\operatorname{ker}\left(\Gamma \rightarrow \Gamma / \Gamma^{m}\right) \cap \operatorname{ker}(\Gamma \rightarrow \Delta)=\Gamma^{m} \cap \operatorname{tor}(\Gamma)=\{e\}
$$

and hence the map $\rho$ is injective. In addition

$$
\operatorname{ker}\left(\Gamma \rightarrow \Delta / \Delta^{m}\right)=\Gamma^{m} \cdot \operatorname{tor}(\Gamma)=\operatorname{ker}\left(\Gamma \rightarrow \Gamma / \Gamma^{m}\right) \cdot \operatorname{ker}(\Gamma \rightarrow \Delta)
$$

and hence, one can check that it follows that $\rho$ is also surjective. Therefore, we can identify $\Gamma$ with $\Delta \times_{\Delta / \Delta^{m}} \Gamma / \Gamma^{m}$, the fiber product of $\Delta$ and $\Gamma / \Gamma^{m}$ along $\Delta / \Delta^{m}$. The following lemma is elementary:

Lemma 3.2. Notation as above. Let $\sigma_{i} \in \operatorname{Aut}\left(P_{i}\right)$ for $i=1,2$ be automorphisms preserving $\operatorname{ker}\left(\pi_{i}\right)$ and inducing $\bar{\sigma}_{i} \in \operatorname{Aut}(Q)$. If $\bar{\sigma}_{1}=\bar{\sigma}_{2}$, then there exists $\sigma \in A$ Aut $(U)$ such that $\sigma$ preseves $U \cap\left(P_{1} \times\{e\}\right)$ and $U \cap\left(\{e\} \times P_{2}\right)$ and induces $\sigma_{1}$ on $P_{1}$ and $\sigma_{2}$ on $P_{2}$.

Proof. For $(x, y) \in U$ define $\sigma(x, y)=\left(\sigma_{1}(x), \sigma_{2}(y)\right)$. We claim that we have $\left(\sigma_{1}(x), \sigma_{2}(y)\right) \in H$. Indeed

$$
\pi_{1}\left(\sigma_{1}(x)\right)=\bar{\sigma}_{1}\left(\pi_{1}(x)\right)=\bar{\sigma}_{1}\left(\pi_{2}(y)\right)=\bar{\sigma}_{2}\left(\pi_{2}(y)\right)=\pi_{2}\left(\sigma_{2}(y)\right) .
$$

Showing that $\sigma$ is a bijective homomorphism follows from the same properties for $\sigma_{1}, \sigma_{2}$. The other properties of $\sigma$ follow straightforward from the definition.

Applying Lemma 3.2 on $\alpha_{m} \in \operatorname{Aut}\left(\Gamma / \Gamma^{m}\right)$ and $\beta \in I A^{*}(\Delta)$ we obtain $\alpha \in$ $\operatorname{Aut}(\Gamma)$ that its projection is $\alpha_{m}$ through $\operatorname{Aut}(\Gamma) \rightarrow \operatorname{Aut}\left(\Gamma / \Gamma^{m}\right)$. Hence, in order to finish the proof of Theorem [1.4, it remains to show that the $\alpha$ we got is not only in $A u t(\Gamma)$ but also in $\alpha \in I A^{*}(\Gamma)$.

Lemma 3.3. Recall that $\Gamma^{*}=\bar{\Gamma} / \operatorname{tor}(\bar{\Gamma})$ where $\bar{\Gamma}=\Gamma / \Gamma^{\prime}$, and denote $\Delta^{*}=$ $\bar{\Delta} / \operatorname{tor}(\bar{\Delta})$ where $\bar{\Delta}=\Delta / \Delta^{\prime}$. Then, we have a canonical isomorphism $\Gamma^{*} \simeq \Delta^{*}$.

Proof. We have a natural projection

$$
\Delta=\Gamma / \operatorname{tor}(\Gamma) \rightarrow \bar{\Gamma} / \operatorname{tor}(\bar{\Gamma})=\Gamma^{*}
$$

which gives rise to a map $\bar{\Delta}=\Delta / \Delta^{\prime} \rightarrow \Gamma^{*}$ and to a map $\Delta^{*}=\bar{\Delta} / \operatorname{tor}(\bar{\Delta}) \rightarrow \Gamma^{*}$. Obviously, this map is the inverse of the natural map $\Gamma^{*} \rightarrow \Delta^{*}$. So $\Gamma^{*} \simeq \Delta^{*}$ as required.

From this lemma we get that the preimage of $I A^{*}(\Delta)$ under the map $A u t(\Gamma) \rightarrow$ $\operatorname{Aut}(\Delta)$ is $I A^{*}(\Gamma)$. Thus, as $\alpha$ is a preimage of $\beta$ through $\operatorname{Aut}(\Gamma) \rightarrow \operatorname{Aut}(\Delta)$ and $\beta \in I A^{*}(\Delta)$ it follows that indeed $\alpha \in I A^{*}(\Gamma)$, as required.

\section{The Free cases}

In this section we sketch a more straightforward proof to Corollary 1.5 in the special case where $\Psi_{c}$ is a free nilpotent group on $n$ elements, a proof which does not refer neither to the CSP nor to the strong approximation for unipotent groups. Notice that as $\Psi_{c}^{*}=\Psi_{c} / \Psi_{c}^{\prime}=\mathbb{Z}^{(n)}$ and $\hat{\Psi}_{c}^{*}=\widehat{\Psi_{c} / \Psi_{c}^{\prime}}=\hat{\mathbb{Z}}^{(n)}$, in this case $I A\left(\Psi_{c}\right)=I A^{*}\left(\Psi_{c}\right)$ and $I A\left(\hat{\Psi}_{c}\right)=I A^{*}\left(\hat{\Psi}_{c}\right)$. Let us formulate the assertion: 
Theorem 4.1. Let $\Psi_{c}$ be the free nilpotent group on $n$ elements. For every $c \in \mathbb{N}$, the map $\widehat{I A\left(\Psi_{c}\right)} \rightarrow I A\left(\hat{\Psi}_{c}\right)$ is an isomorphism.

Proof. We will prove it by induction on $c$. For $c=1,2$ the result is trivial, as $\Psi_{1}=\{e\}$ and $\Psi_{2}=\mathbb{Z}^{(n)}$, so

$$
\left.\widehat{I A\left(\Psi_{1}\right)}=I A\left(\hat{\Psi}_{1}\right)=\widehat{I A\left(\Psi_{2}\right.}\right)=I A\left(\hat{\Psi}_{2}\right)=\{e\} .
$$

For the induction step we will use the (easy) facts that for every $c$, the natural map $\operatorname{Aut}\left(\Psi_{c+1}\right) \rightarrow \operatorname{Aut}\left(\Psi_{c}\right)$ is surjective (see [A]), and the natural map $\operatorname{Aut}\left(\hat{\Psi}_{c+1}\right) \rightarrow \operatorname{Aut}\left(\hat{\Psi}_{c}\right)$ is also surjective (see Section 5.2 in [L2]). So let $c \geq 2$. Denote

$$
\begin{aligned}
& \left.A\left(\Psi_{c+1}\right)=\operatorname{ker}\left(I A\left(\Psi_{c+1}\right)\right) \rightarrow I A\left(\Psi_{c}\right)\right) \\
& \left.A\left(\hat{\Psi}_{c+1}\right)=\operatorname{ker}\left(I A\left(\hat{\Psi}_{c+1}\right)\right) \rightarrow I A\left(\hat{\Psi}_{c}\right)\right) .
\end{aligned}
$$

We have the commutative exact diagram

$$
\begin{aligned}
& 1 \rightarrow A\left(\Psi_{c+1}\right) \rightarrow I A\left(\Psi_{c+1}\right) \rightarrow I A\left(\Psi_{c}\right) \rightarrow 1 \\
& \begin{array}{c}
\downarrow \\
1 \rightarrow A\left(\hat{\Psi}_{c+1}\right)
\end{array} \rightarrow I A\left(\hat{\Psi}_{c+1}\right) \rightarrow \underset{ }{\downarrow} \rightarrow\left(\hat{\Psi}_{c}\right) \rightarrow 1
\end{aligned}
$$

which gives rise to the commutative exact diagram

$$
\begin{aligned}
& \widehat{A\left(\Psi_{c+1}\right)} \rightarrow I \widehat{A\left(\Psi_{c+1}\right)} \rightarrow \widehat{I A\left(\Psi_{c}\right)} \rightarrow 1 \\
& 1 \rightarrow A\left(\hat{\Psi}_{c+1}^{\downarrow}\right) \rightarrow I A\left(\hat{\Psi}_{c+1}\right) \rightarrow \begin{array}{c}
\downarrow \\
I A\left(\hat{\Psi}_{c}\right)
\end{array} \rightarrow 1 .
\end{aligned}
$$

By the induction hypothesis and diagram chasing, it is enough to prove that the map $\widehat{A\left(\Psi_{c+1}\right)} \rightarrow A\left(\hat{\Psi}_{c+1}\right)$ is an isomorphism.

Let $x_{1}, \ldots, x_{n}$ be a set of free generators for $\Psi_{c+1}$. Denote

$$
Z\left(\Psi_{c+1}\right)^{(n)}=\underbrace{Z\left(\Psi_{c+1}\right) \times \ldots \times Z\left(\Psi_{c+1}\right)}_{n} .
$$

where $Z\left(\Psi_{c+1}\right)$ is the center of $\Psi_{c+1}$. Observe now that $A\left(\Psi_{c+1}\right)$ can be viewed as a subgroup of $Z\left(\Psi_{c+1}\right)^{(n)}$ in the following way: For $\alpha \in A\left(\Psi_{c+1}\right)$, one can describe it by its action on the generators of $\Psi_{c+1}$ and write $\alpha\left(x_{i}\right)=x_{i} u_{i}$ for some $u_{i} \in Z\left(\Psi_{c+1}\right)$. We claim that the map $\alpha \mapsto\left(u_{1}, \ldots, u_{n}\right) \in Z\left(\Psi_{c+1}\right)^{(n)}$ is a natural injective homomorphism $A\left(\Psi_{c+1}\right) \rightarrow Z\left(\Psi_{c+1}\right)^{(n)}$. Indeed, let $\alpha, \beta \in$ $A\left(\Psi_{c+1}\right)$ defined by $\alpha\left(x_{i}\right)=x_{i} u_{i}$ and $\beta\left(x_{i}\right)=x_{i} v_{i}$ for some $u_{i}, v_{i} \in Z\left(\Psi_{c+1}\right)$. Now, as $u_{i} \in Z\left(\Psi_{c+1}\right) \subseteq \Psi_{c+1}^{\prime}$ it can be written as a product of commutators of words on the generators $x_{i}$. As $u_{i}, v_{i} \in Z\left(\Psi_{c+1}\right)$, it is easy to see that $(\beta \circ \alpha)\left(x_{i}\right)=x_{i} v_{i} u_{i}$. This proves the claim. In [A] it is proven that the above map $A\left(\Psi_{c+1}\right) \rightarrow Z\left(\Psi_{c+1}\right)^{(n)}$ is also surjective, and hence, it is an isomorphism. I.e., for every choice of elements $u_{1}, \ldots, u_{n} \in Z\left(\Psi_{c+1}\right)$, one can define an element $\alpha \in A\left(\Psi_{c+1}\right)$ by defining $\alpha\left(x_{i}\right)=x_{i} u_{i}$ for the generators $x_{1}, \ldots, x_{n}$ of $\Psi_{c+1}$. 
This is indeed an automorphism as $\left\{x_{i} u_{i}\right\}_{i=1}^{n}$ generate $\Psi_{c+1}$, which is a free nilpotent group.

Using a similar approach, one can prove that $A\left(\hat{\Psi}_{c+1}\right) \cong Z\left(\hat{\Psi}_{c+1}\right)^{(n)}$ (see also [L2]) and thus

$$
\widehat{A\left(\Psi_{c+1}\right)} \cong Z{\widehat{\left(\Psi_{c+1}\right)}}^{(n)} \cong Z\left(\hat{\Psi}_{c+1}\right)^{(n)} \cong A\left(\hat{\Psi}_{c+1}\right)
$$

as required.

\section{References}

[A] S. Andreadakis, On the automorphisms of free groups and free nilpotent groups, Proc. London Math. Soc. 15 (1965), 239-268.

[Ba] G. Baumslag, Automorphism groups of nilpotent groups. Amer. J. Math. 91 (1969), 1003-1011.

[Be1] D. E-C. Ben-Ezra, The congruence subgroup problem for the free metabelian group on two generators. Groups Geom. Dyn. 10 (2016), $583-599$.

[Be2] D. E-C. Ben-Ezra, The IA-congruence kernel of high rank free Metabelian groups, Annals of K-Theory 4 (2019), 383-438.

[Be3] D. E-C. Ben-Ezra, The congruence subgroup problem for the free metabelian group on $n \geq 4$ generators, Groups Geom. Dyn. to appear, arXiv:1701.02459

[BER] K-U. Bux, M. V. Ershov, A. S. Rapinchuk, The congruence subgroup property for Aut $\left(F_{2}\right)$ : a group-theoretic proof of Asada's theorem, Groups Geom. Dyn. 5 (2011), 327-353.

[BG] R. M. Bryant, J. R. J. Groves, Algebraic groups of automorphisms of nilpotent groups and Lie algebras, Journal of the London Mathematical Society 2 (1986), 453-466.

[BP] R. M. Bryant, A. Papistas, Automorphism groups of nilpotent groups, Bulletin of the London Mathematical Society 21 (1989), 459-462.

[BL] D. E-C. Ben-Ezra, A. Lubotzky, The congruence subgroup problem for low rank free and free metabelian groups, J. Algebra 500 (2018), 171-192.

[BLS] H. Bass, M. Lazard, J.-P. Serre, Sous-groupes d'indice fini dans $S L_{n}(\mathbb{Z})$, Bull. Amer. Math. Soc. 70 (1964) 385-392 (French).

[C] J. S. Chahal, Solution of the congruence subgroup problem for solvable algebraic groups, Nagoya Mathematical Journal 79 (1980) 141-144. 
[DDSM] J. D. Dixon, M. P. Du Sautoy, D. Segal, A. Mann, Analytic pro-p groups, Cambridge University Press, 2003.

[KW] P. H. Kropholler, J. S. Wilson, Torsion in profinite completions, J. Pure Appl. Algebra, 88 (1993) 143-154.

[L1] A. Lubotzky, Free quotients and the congruence kernel of $S L_{2}$, J. Algebra 77 (1982), 411-418.

[L2] A. Lubotzky, Combinatorial group theory for pro- $p$-groups, J. Pure Appl. Algebra 25 (1982), 311-325.

[Mel] O. V. Mel'nikov, Congruence kernel of the group $S L_{2}(\mathbb{Z})$, (Russian) Dokl. Akad. Nauk SSSR 228 (1976), 1034-1036.

[Men] J. L. Mennicke, Finite factor groups of the unimodular group, Ann. of Math. 81 (1965), 31-37.

[NS] N. Nikolov, D. Segal, Finite index subgroups in profinite groups, C. R. Math. Acad. Sci. Paris 337 (2003), 303-308.

[P] V. P. Platonov, On the congruence subgroup problem for integral soluble groups, Dokl. Akad. Nauk BSSR 15 (1971), 869-872 (Russian).

[PS] V. P. Platonov, A. A. Sharonet, On the congruence subgroup problem for linear groups over arithmetic rings, Dokl. Akad. Nauk BSSR 16 (1972), 393-396 (Russian).

[PR] V. Platonov, A. Rapinchuk, Algebraic Groups and Number Theory, Academic press, 1993.

[Rag] M. S. Raghunathan, The congruence subgroup problem, Proc. Indian Acad. Sci. Math. Sci. 114 (2004), 299-308.

[Rap] A. S. Rapinchuk, The congruence subgroup problem, Algebra, Ktheory, Groups, and Education, New York, 1997, 175-188.

[S] D. Segal, Polycyclic Groups, No. 82, Cambridge University Press, 2005.

[W] R. B. Warfield Jr. Nilpotent groups. Lecture Notes in Mathematics, Vol. 513, Springer-Verlag, Berlin-New York, 1976.

Ben-Ezra, David El-Chai

Department of Mathematics

University of California in San-Diego

La Jolla, CA 92093

USA

davidel-chai.ben-ezra@mail.huji.ac.il
Lubotzky, Alexander

Einstein Institute of Mathematics

The Hebrew University

Jerusalem, 91904

Israel

alex.lubotzky@mail.huji.ac.il 